\title{
Impacto de los servicios de salud, el saneamiento y la alfabetización en la mortalidad de menores de cinco años
}

Gonzalo Gutiérrez, M.C., M.S.P., ${ }^{(1)}$ H ortensia Reyes, M.C., M. en C., (1) Sonia Fernández, Act., Ph.D., (2) Luis Pérez, L. en Admón., (1) Ricardo Pérez-C uevas, M.C., M. en C., M.H. Sc., ${ }^{(1)}$ Héctor G uiscafré, M.C., M. en C. (1)

\author{
Gutiérrez G, Reyes H, Fernández S, \\ Pérez L, Pérez-Cuevas R, Guiscafré $H$. \\ Impacto de los servicios de salud, \\ el saneamiento y la educación \\ en la mortalidad de menores de cinco años. \\ Salud Publica Mex 1999;41:368-375.
}

\section{Resumen}

Objetivo. A nalizar el impacto de los servicios de salud, el saneamiento y la alfabetización en la mortalidad de menores de cinco años en dos estados con diferentes características socioculturales: Chiapas y Nuevo León. Material y métodos. Se realizó un estudio ecológico con base en el análisis secundario de datos publicados por la Secretaría de Salud, el Instituto $N$ acional de Estadística, Geografía e Informática y el Consejo $\mathrm{N}$ acional de Población, relativos a las tendencias de la mortalidad de menores de cinco años y a cambios de diversos indicadores, correspondientes al periodo de 1990-1997. Mencionar al tratamiento estadístico de los datos. Se realizó un estudio ecológico con base en el análisis secundario de datos publicados por la Secretaría de Salud, el Instituto $\mathrm{N}$ acional de Estadística e Informática y el Consejo $\mathrm{N}$ acional de Población, relativos a las tendencias de MMCA y a cambios de diversos indicadores, correspondientes al periodo de 1990-1997. Para ambos estados se calcularon las variaciones registradas y se determinaron las tendencias mediante análisis de regresión lineal simple, en el que la variable independiente correspondió a los años de estudio. Se hizo también análisis de correlación parcial entre las diversas tendencias de mortalidad estudiadas y entre éstas y los indicadores seleccionados. Resultados En el periodo estudiado se registró un marcado descenso de la mortalidad de menores de cinco años que fue más acentuado en Chiapas; en ambas entidades se relacionó principalmente con la disminución de mortalidad por enfermedad diarreica, aunque también con la de sarampión e infecciones respiratorias agudas. En C hiapas los indicadores
Gutiérrez G, Reyes H, Fernández S, Pérez L, Pérez-Cuevas R, Guiscafré H. Impact of health care services, sanitation and education on mortality rates of children under five years of age. Salud Publica Mex 1999;41:368-375.

\begin{abstract}
A bstract
Objective. To analyze differences of the impact of health care services, sanitation and literacy on the mortality rates of children under five years of age, in two Mexican states with marked socioeconomic differences: $\mathrm{C}$ hiapas and $\mathrm{N}$ uevo Leon. Material and methods The study design was ecologic, based on a retrospective analysis of data published by the Health Ministry (Secretaría de Salud), N ational Institute of Statistics, G eography and Informatics (Instituto $\mathrm{N}$ acional de Estadística, Geografía e Informática) and the $\mathrm{N}$ ational Population Council (Consejo Nacional de Población), on the tendencies of mortality among children under five years and on the changes of selected indicators corresponding to the period 1990-1997. Study design: ecologic study. This was based on a retrospective analysis of data published by Secretaría de Salud, Instituto $\mathrm{N}$ acional de Estadística e Informática and Consejo $\mathrm{N}$ acional de Población, about the tendencies of mortality among children under five years, and about the changes of selected indicators. The analysis was carried out in the period comprised between 1990-1997. For both states the registered variations were calculated and the trends were determined through analysis of simple linear regression; the independent variable corresponded to the study years. Partial correlation analysis between the various mortality trends studies and between and the selected indicators, were calculated. Results. During the studied period there was a steady decline of children mortality, which was more marked in Chiapas. In both entities, this decrease was closely related to the decline in mortality due to acute diarrhea, and also correlated
\end{abstract}

(1) Grupo Interinstitucional de Investigación en Sistemas de Salud, Secretaría de Salud e Instituto Mexicano del Seguro Social, México.

(2) Dirección General de Estadística e Informática, Secretaría de Salud, México.

Fecha de recibido: 21 de enero de 1999 - Fecha de aprobado: 7 de mayo de 1999 Solicitud de sobretiros: Gonzalo Gutiérrez. Hortensia 57, colonia Florida, 01030 México, D.F., México. Correo electrónico: farah@ mail.internet.com.mx 
que correlacionaron más significativamente con este descenso fueron las coberturas de vacunación y el alfabetismo; en $\mathrm{N}$ uevo León, el aumento en el número de enfermeras, las coberturas de vacunación, el alfabetismo y las viviendas con agua entubada. Conclusiones En el periodo estudiado se redujo el diferencial en las tasas de mortalidad de menores de cinco años entre Chiapas y N uevo León; para mantener 0 acelerar tal descenso es necesario continuar con los programas analizados y, en Chiapas o estados similares, ampliar en forma más acelerada la infraestructura en salud.

Palabras clave:mortalidad menores cinco años; impacto programas salud; enfermedad diarreica; infección respiratoria aguda; sarampión with a descent in measles and acute respiratory infections. In C hiapas, the indicators which correlated more significantly with this decline in mortality were vaccination coverage and literacy. In N uevo Leon, the indicators with greater correlation were the increase in the number of nurses, of lodgings with piped water and vaccination coverage. Conclusions During the analyzed period, the mortality rate of children under five years of age decreased in the states of Chiapas and N uevo Leon.To sustain or accelerate the decline in childhood mortality it is mandatory to continue with the currently implemented programs, and in Chiapas, and similar states, to increase the available infrastructure to provide health care.

Key words: mortality less five years old; impact of health programs; diarrhoeal disease; acute respiratory infection; measles as diferencias culturales, sociales y económicas que en México persisten entre muy diversas regiones, tienen orígenes y manifestaciones complejas, con frecuencia ambivalentes: por un lado, se puede apreciar la riqueza de la diversidad cultural existente pero, por otro, están los rezagos económicos y sociales, entre los que destacan aquellos relacionados con la salud, tanto en la prestación de servicios como en la salud misma de las poblaciones. ${ }^{1}$ Las políticas, los programas y las acciones para corregir o atenuar estos rezagos y desigualdades, aunque insuficientes, han sido constantes pero no siempre evaluados. En este sentido, la mortalidad en niños menores de cinco años es un indicador muy sensible -e internacionalmente aceptado-, para medir la sobrevivencia, el bienestar y el desarrollo de la infancia, así como el impacto de los programas de salud, educación, cuidado ambiental y asistencia social; puede decirse que, en general, sirve para medir el desarrollo socioeconómico. ${ }^{2}$

A partir de 1990 diversos programas de salud, educación y saneamiento recibieron en México un importante impulso, debido principalmente a dos circunstancias: a) al arribo de las pandemias de sarampión y cólera y b) a los compromisos internacionales que nuestro país adquirió para la erradicación, la eliminación o el control de diversas enfermedades, fundamentalmente de aquellas prevenibles por vacunación, de las enfermedades diarreicas y de las infecciones respiratorias agudas en la infancia..$^{3-5}$ Simultáneamente, la ampliación de los servicios de salud, de saneamiento básico y de educación continuó avanzando en todo el país. $^{6}$

El propósito de este trabajo es evaluar el impacto de los servicios antes mencionados, mediante el análisis de las tendencias de la mortalidad en los menores de cinco años y su correlación con diversos indicadores de salud, saneamiento y educación, en dos estados de la República mexicana: Chiapas y Nuevo León. Se trata de dos entidades que, teniendo un volumen poblacional semejante, históricamente han mostrado las mayores diferencias socioeconómicas y sanitarias entre sí, además de que en 1990 registraron los valores extremos de mortalidad en menores de cinco años, en el ámbito nacional. Cabe añadir que, de acuerdo con el XI Censo General de Población y Vivienda, Chiapas tiene una elevada proporción de población rural $(76.5 \%)$ e indígena $(26.4 \%)$, mientras que en Nuevo León, estado predominantemente urbano $(87.2 \%)$, sólo $0.2 \%$ de la población habla alguna lengua indígena. ${ }^{7}$

\section{Material y métodos}

Se realizó un estudio ecológico con base en el análisis secundario de datos publicados en los Anuarios Estadísticos de la Secretaría de Salud (SSA), ${ }^{8}$ en los boletines de la Dirección General de Epidemiología ${ }^{9}$ y del Consejo Nacional de Vacunación, ${ }^{10,11}$ así como en las publicaciones del Instituto Nacional de Estadística, Geografía e Informática (INEGI) ${ }^{7,12,13}$ y del Consejo Nacional de Población (CONAPO).$^{14}$

En la selección de las series de datos utilizados se tomó en cuenta la calidad y la confiabilidad de los mismos durante el periodo analizado, de tal forma que se eliminaron los menos sólidos. En el caso de la mortalidad por todas las causas en menores de cinco años, se utilizaron las tasas ajustadas para corregir el subregistro, como se menciona a continuación.

Se analizó la información anual del periodo 1990 1997, relativa a la mortalidad en menores de cinco años, 
utilizando también la IX Clasificación Internacional de Enfermedades:

- Mortalidad ajustada por subregistro (todas las causas), por cada 1000 nacidos vivos esperados. Para la estimación del subregistro de muertes en los menores de un año se calculó la mortalidad infantil con el método de Brass, ${ }^{15}$ según los datos del Censo de 1990, y con base en ello se obtuvo un factor de subregistro que fue aplicado a cada año de referencia. El subregistro en los niños de 1 a 4 años se estimó considerando otro factor, notificado por la Encuesta Nacional de la Dinámica Demográfica, hecha por el INEGI en 1992. Por último se tomó como denominador la cifra de nacimientos esperados estimada por el CONAPO de acuerdo con la tendencia de la fecundidad.

- Mortalidad por sarampión (E042), por cada 100000 habitantes del grupo de edad.

- Mortalidad por enfermedades diarreicas (E01) y por infecciones respiratorias agudas (E310, E312, E320, E321 y E322), por 100000 habitantes del grupo de edad.

Para el mismo periodo, también se analizaron indicadores anuales relativos a:

1. Servicios de salud: a) coberturas de vacunación en niños de 1 a 4 años, con esquemas completos del Programa Ampliado de Inmunizaciones (PAI): Sabin (tres dosis) DPT (tres dosis) BCG y antisarampión; b) número de médicos por cada 100000 habitantes, y c) número de enfermeras por cada 100000 habitantes.

2. Saneamiento: a) porcentaje de viviendas con drenaje, y b) porcentaje de viviendas con agua entubada.

3. Educación: a) porcentaje de alfabetas mayores de 15 años de edad.

La serie anual de los indicadores de saneamiento y educación se construyó con base en los datos del Censo General de Población y Vivienda de 1990 y del Conteo de Población y Vivienda de 1995.

\section{A nálisis}

Para ambos estados se calcularon las variaciones registradas entre 1990 y 1997, tanto en la mortalidad en menores de cinco años, como en los indicadores seleccionados. Se determinaron las tendencias en este mismo periodo mediante análisis de regresión lineal simple, en el que la variable independiente correspondió a los años de estudio.

Se efectuó, además, un análisis de correlación parcial entre las diversas tasas de mortalidad estudiadas, por un lado, y entre las tasas de mortalidad y los indicadores seleccionados, por el otro, lo que permitió ajustar en cada variable de interés el peso del resto de los indicadores. Se calculó también el valor de $p$ en cada una de las correlaciones analizadas y se le consideró significativo cuando fue $<0.05$.

\section{Resultados}

Entre 1990 y 1997 las tasas de mortalidad entre los menores de cinco años disminuyeron en todo México, y aunque las tendencias negativas fueron estadísticamente significativas, tuvieron características diferentes en los estados comparados: en Chiapas el descenso fue mayor que en Nuevo León. En el primer caso, la tasa ajustada de mortalidad por todas las causas disminuyó 40.6 puntos, y la de sarampión, 137.8; la de enfermedades diarreicas, 201.9, y la de infecciones respiratorias agudas, 47.3 En Nuevo León el descenso en cada una de ellas fue de 5.8, 8.0, 35.2 y 33.2 puntos, respectivamente. Consecuentemente las brechas entre los dos estados, en las cuatro tendencias, se acortaron significativamente en el periodo estudiado (figuras 1 y 2).

En el mismo lapso, los indicadores de salud, saneamiento y educación, mejoraron en todo el país, pero también se registraron diferencias entre los dos estados comparados. En Chiapas, el incremento de las coberturas de vacunación, de viviendas con agua y drenaje, y de alfabetismo fue mayor que en Nuevo León, aunque se registró una situación contraria en lo que respecta al aumento del número de médicos y de enfermeras. Consecuentemente, se acortaron las brechas relacionadas con los primeros indicadores; en cambio, aquellas que tienen que ver con el número de médicos y enfermeras inclusive se ampliaron (cuadro I y figura 3).

El cálculo de correlaciones y de su significación estadística también mostró diferencias entre Chiapas y Nuevo León (cuadro II).

La correlación entre la tendencia de la mortalidad por todas las causas (ajustada por subregistro) y la de las tres mortalidades específicas analizadas, fue muy elevada y estadísticamente significativa en los dos estados $(p<0.01)$. La relativa a enfermedades diarreicas se ubicó en el primer lugar, en las dos entidades federativas. Las otras dos causas analizadas se ordenaron en forma diferente: en Chiapas la correlación más 


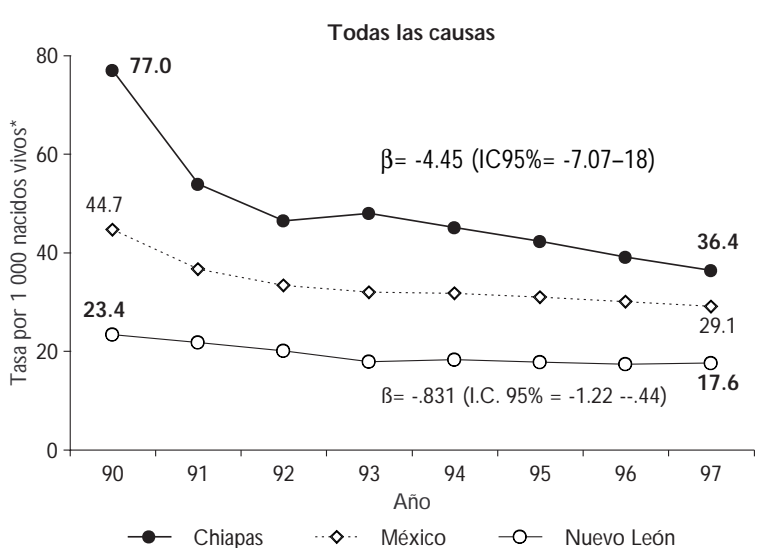

* Ajustada por subregistro

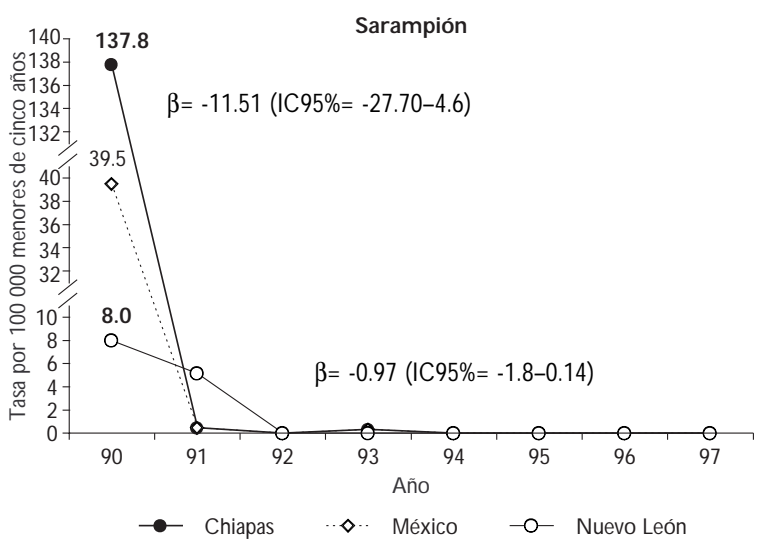

Fuente: Dirección General de Estadística e Informática, Secretaría de Salud, México

Figura 1. Mortalidad en menores de cinco años. Chiapas y Nuevo León, México 1990-1997

elevada fue con la mortalidad por sarampión, y en Nuevo León, con la de infecciones respiratorias agudas.

Las correlaciones entre las tendencias de la mortalidad y los indicadores de servicios de salud, saneamiento y educación mostraron las siguientes diferencias:

1. En Chiapas (cuadro II) las tendencias de la mortalidad por todas las causas, por sarampión y por infecciones respiratorias agudas, al ajustar por el resto de los indicadores, presentaron correlación negativa y estadísticamente significativa sólo con las coberturas de vacunación. La tendencia de la mortalidad por enfermedades diarreicas tuvo correlación negativa significativa
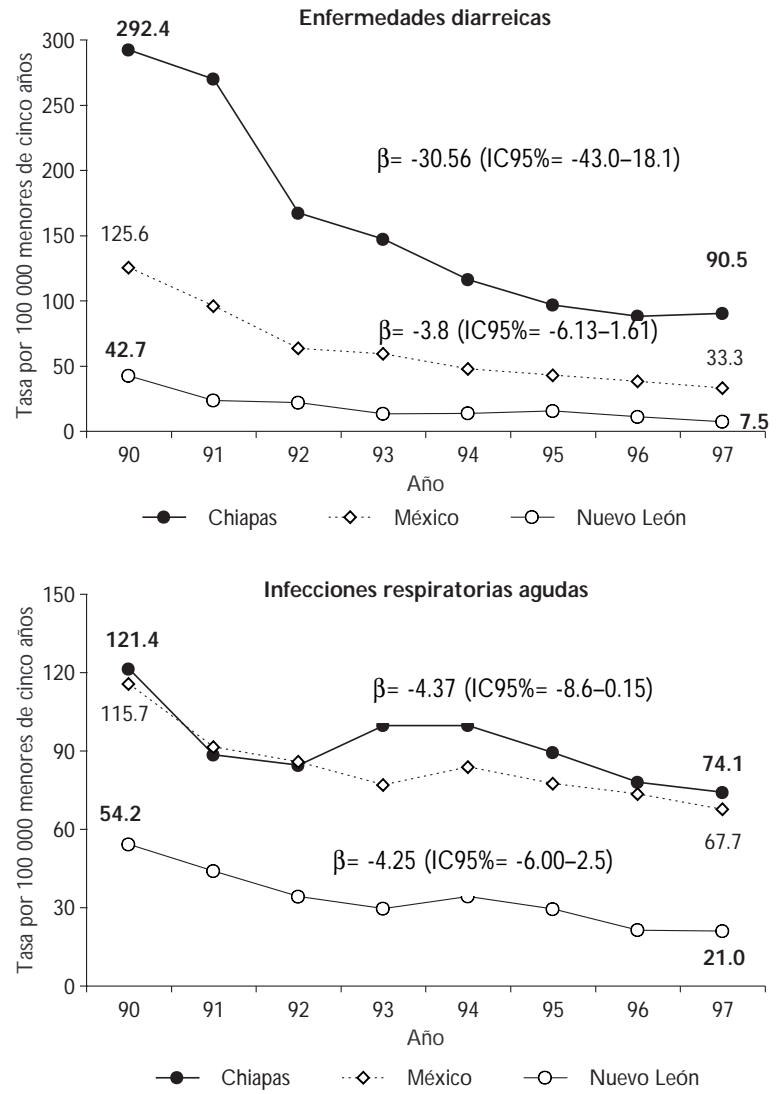

Fuente: Dirección General de Estadística e Informática, Secretaría de Salud, México

Figura 2. Mortalidad en MENORES de Cinco años por ENFERMEDADES DIARREICAS E INFECCIONES RESPIRATORIAS agudas. Chiapas y Nuevo León, México, 1990-1997

con alfabetismo y también con coberturas de vacunación.

2. En Nuevo León (cuadro II) las tendencias de la mortalidad por todas las causas y por infecciones respiratorias agudas se correlacionaron significativamente con las del número de enfermeras. La tendencia de la mortalidad por enfermedades diarreicas mostró correlación negativa principalmente con la de coberturas de vacunación y, en segundo término, con la proporción de viviendas con agua entubada. La de la mortalidad por sarampión correlacionó con tres indicadores, que fueron, en orden de importancia, el número de enfermeras, el alfabetismo y la proporción de viviendas con agua entubada. 


\section{Cuadro I \\ VARIACIÓN DE LOS INDICADORES SELECCIONADOS PARA ESTIMAR EL IMPACTO DE LOS SERVICIOS DE SALUD en la mortalidad de menores de cinco años. Chiapas y Nuevo León, México, 1990-1997}

\begin{tabular}{|c|c|c|c|c|c|c|c|c|c|}
\hline \multirow{4}{*}{ Coberturas de vacunación en niños } & \multicolumn{3}{|c|}{ México } & \multicolumn{3}{|c|}{ Chiapas } & \multicolumn{3}{|c|}{ Nuevo León } \\
\hline & 1990 & 1997 & rencia 90-97 & 1990 & 1997 & rencia 90-97 & 1990 & 1997 & encia 90-97 \\
\hline & & & & & & & & & \\
\hline & 46.0 & 96.8 & 50.8 & 15.8 & 93.2 & 77.4 & 55.1 & 98.8 & 43.7 \\
\hline N úmero de médicos* & & & & & & & & & \\
\hline Tasa por 100000 habitantes & 93 & 116 & 23 & 47 & 83 & 36 & 86 & 130 & 44 \\
\hline N úmero de enfermeras* & & & & & & & & & \\
\hline Tasa por 100000 habitantes & 160 & 182 & 22 & 84 & 112 & 28 & 189 & 257 & 68 \\
\hline Viviendas con drenaje (\%) & 63.6 & 79.2 & 15.6 & 41.2 & 61.9 & 20.7 & 80.9 & 91.7 & 10.8 \\
\hline Viviendas con agua entubada (\%) & 75.5 & 89.7 & 14.2 & 58.4 & 70.1 & 11.7 & 92.6 & 94.9 & 2.3 \\
\hline Alfabetismo en mayores de 15 años (\%) & 87.6 & 90.1 & 2.5 & 70.0 & 75.6 & 5.6 & 95.4 & 96.5 & 1.1 \\
\hline
\end{tabular}

\section{Discusión}

Durante las últimas dos décadas y principalmente en los países con elevada mortalidad, se han realizado múltiples estudios para evaluar intervenciones en salud, con el interés de desarrollar y evaluar estrategias efectivas para la aplicación de diversos programas. Sin embargo, y a pesar de que se ha demostrado la eficacia de diversos ensayos de campo, aún existe debate en torno a los programas derivados de resultados obtenidos en condiciones controladas, así como respecto a su aplicación a gran escala o en poblaciones con situaciones particulares de riesgo. La mortalidad no ocurre aleatoriamente en una población; depende de condiciones sociodemográficas, ambientales, reproductivas y de nutrición, así como de la cobertura y la calidad de la atención a la salud. ${ }^{16}$ Por esta razón, evaluar los posibles impactos de los programas de salud requiere de un enfoque que incluya el contexto de la población a la que fueron dirigidos. Esta fue la premisa del presente estudio.

Para la apropiada interpretación de los resultados es necesario considerar previamente algunos aspectos relevantes, derivados del diseño seleccionado. En primer lugar, debe aceptarse que los análisis de fuentes secundarias de datos sólo permiten explorar de manera muy general la realidad, lo que no da la pauta para establecer relaciones directas entre las variables de estudio; sin embargo, brindan la posibilidad de ge- nerar hipótesis con un sustento sólido, cuando éstas están basadas en indicadores cuidadosamente elegidos con este fin. En segundo término, se ha documentado que la información derivada de registros estadísticos está expuesta a errores; uno de los más relevantes es el subregistro de las estadísticas vitales. ${ }^{17,18}$ En este trabajo, se decidió utilizar los datos de mortalidad por todas las causas en menores de cinco años, ajustada por subregistro y con métodos que han demostrado ser confiables. ${ }^{19}$

Dicho lo anterior, es necesario destacar en primer lugar que, excepto en poblaciones con pobreza extrema o pasando por momentos de crisis sociales muy severas, la tendencia mundial de la mortalidad en la infancia, en los últimos años, ha sido descendente. ${ }^{2}$ México no ha sido la excepción; más aún, el Fondo de las Naciones Unidas para la Infancia lo ubica entre los países que más rápidamente están avanzando en la reducción de la mortalidad de los menores de cinco años. ${ }^{2}$ Entre 1990 y 1997, la tasa anual por 1000 nacidos vivos disminuyó significativamente (34.9\%), pero este descenso no fue homogéneo en todo el país: fue más acelerado en los estados con tasas de mortalidad más altas y con mayores rezagos socioeconómicos y en salud; consecuentemente, la brecha que en este sentido existe entre entidades con diferente nivel de desarrollo disminuyó. ${ }^{6}$ El ejemplo más ilustrativo es el de los estados de Chiapas y Nuevo León, que en 1990 registraron, respectivamente, la mayor y menor tasa 


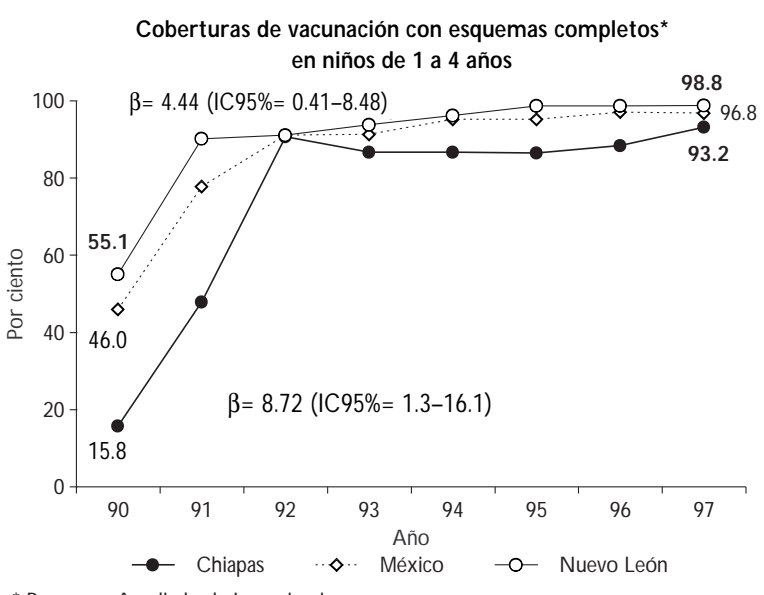

* Programa Ampliado de Inmunizaciones
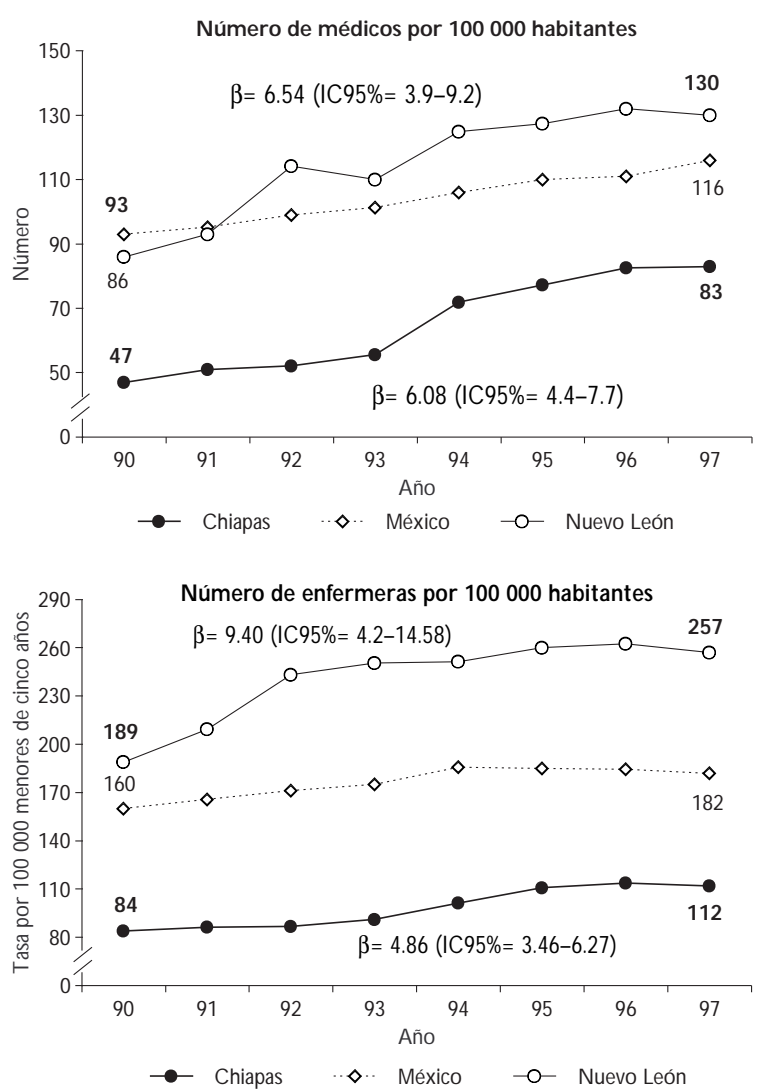

Fuente: Consejo N acional de Vacunación.

Dirección General de Estadística e Informática, Secretaría de Salud, México

Figura 3. Evolución de algunos indicadores de servicios de salud. Chiapas y Nuevo León, México, 1990-1997

\section{Cuadro II \\ Correlación entre la mortalidad en menores \\ DE CINCO AÑOS Y LOS INDICADORES SELECCIONADOS. Chiapas y Nuevo León, México, 1990-1997}

$\begin{array}{ccc}\text { M ortalidad/indicadores* Chiapas } & \text { Correlación } & \text { Valor } \\ \text { parcial } & \text { de } p\end{array}$

Mortalidad por todas las causas

\begin{tabular}{lll} 
Enfermedades diarreicas & 0.985 & 0.000 \\
\hline Sarampión & 0.959 & 0.002 \\
\hline Infecciones respiratorias agudas & 0.945 & 0.004 \\
\hline Coberturas de vacunación & 0.940 & 0.001
\end{tabular}

Mortalidad por enfermedades diarreicas

$\begin{array}{lll}\text { Alfabetismo en mayores de } 15 \text { años } & 0.946 & 0.005\end{array}$

$\begin{array}{lll}\text { Coberturas de vacunación } & 0.829 & 0.020\end{array}$

Mortalidad por sarampión

$\begin{array}{lll}\text { Coberturas de vacunación } & 0.854 & 0.007\end{array}$

Mortalidad por infecciones respiratorias agudas

Coberturas de vacunación

$0.734 \quad 0.038$

Nuevo León

Mortalidad por todas las causas

\begin{tabular}{lll} 
Enfermedades diarreicas & 0.917 & 0.000 \\
\hline Infecciones respiratorias agudas & 0.870 & 0.000 \\
\hline Sarampión & 0.591 & 0.004 \\
\hline N úmero de enfermeras & 0.979 & 0.000
\end{tabular}

Mortalidad por enfermedades diarreicas

$\begin{array}{lll}\text { Coberturas de vacunación } & 0.922 & 0.003\end{array}$

$\begin{array}{lll}\text { Viviendas con agua entubada } & 0.787 & 0.036\end{array}$

Mortalidad por infecciones respiratorias agudas

$\begin{array}{lll}N \text { úmero de enfermeras } & 0.953 & 0.003\end{array}$

Mortalidad por sarampión

$\begin{array}{lll}\mathrm{N} \text { úmero de enfermeras } & 0.971 & 0.000\end{array}$

$\begin{array}{lll}\text { Alfabetismo en mayores de } 15 \text { años } & 0.789 & 0.020\end{array}$

$\begin{array}{lll}\text { Viviendas con agua entubada } & 0.767 & 0.013\end{array}$

* Se anotan solamente los indicadores que correlacionaron significativamente

de mortalidad de menores de cinco años: en el primer caso, ésta fue 300.3\% más elevada. En 1997, aunque todavía fue significativamente mayor, la diferencia se redujo a $100.1 \%$.

Durante el periodo analizado, se registró en México una disminución general de todas las causas de la 
mortalidad de menores de cinco años, pero las que más contribuyeron a este descenso fueron las enfermedades diarreicas, el sarampión y las infecciones respiratorias agudas. ${ }^{8}$ El fenómeno fue similar en Chiapas y Nuevo León; de acuerdo con el análisis hecho en el presente trabajo, las tendencias de las tres causas de muerte mencionadas mostraron una correlación muy significativa con la tendencia de la mortalidad por todas las causas en los dos estados estudiados, aunque con un ordenamiento interesante que merece ser comentado.

Destaca que en ambas entidades la mayor correlación se estableció con la enfermedad diarreica. Debe recordarse que en este periodo se dio gran énfasis al Programa de Prevención y Control de Enfermedades Diarreicas -que se basa en la promoción de la terapia de hidratación oral y en la capacitación de las madres-, así como al Programa "Agua Limpia" -cuyo objetivo principal es mejorar y ampliar los sistemas de cloración del agua-; ambos fueron evaluados en un trabajo previo, de tal forma que se demostró su impacto en la reducción de la mortalidad por enfermedad diarreica. ${ }^{20}$ Asimismo, la mayor correlación de la mortalidad en menores de cinco años con la disminución de la mortalidad por sarampión en Chiapas, quizá se deba a que la pandemia de 1990 originó tasas de mortalidad muy elevadas en este estado, que rápidamente descendieron ${ }^{8}$ gracias a la vacunación con altas coberturas, a lo largo de todo el periodo analizado. ${ }^{11} \mathrm{En}$ Nuevo León, la tasa de mortalidad de 1990 fue mucho menor y, por lo tanto, el descenso fue también menor. Se ha demostrado que en este tipo de enfermedades el resultado de una intervención eficaz y efectiva puede ser observado a corto plazo, y en el caso del sarampión, la efectividad de la vacuna puede ser mayor a 95\%. ${ }^{21}$ En cambio, en Nuevo León, donde la disminución de la mortalidad en menores de cinco años tuvo mayor correlación con la tendencia de la mortalidad por infecciones respiratorias agudas, muy probablemente ello estuvo relacionado con el mejor acceso y calidad de los servicios de salud; estudios previos de nuestro grupo han encontrado que el proceso de atención médica tiene un mayor peso en la mortalidad por este padecimiento que las condiciones biológicas o del medio ambiente. ${ }^{22}$

Existen modelos causales en los que se propone la influencia de distintos factores sobre la mortalidad y la supervivencia de los menores de cinco años. Entre ellos destacan diversos aspectos biomédicos como la interacción desnutrición-infección, así como aquellos relacionados con el cuidado de los niños en el hogar, la atención médica y con diversas condiciones sanitarias y socioeconómicas, cuya influencia en la morta- lidad varía en relación con el tiempo de exposición a dichos factores. ${ }^{23}$ En los dos estados analizados, los orígenes del acelerado descenso de la mortalidad en menores de cinco años en el último decenio se ubican especialmente entre los factores que la pueden modificar en el corto o mediano plazo. Los de naturaleza cultural y socioeconómica, en especial los estilos de vida, la alimentación y los patrones de asentamiento urbano o rural, sólo pueden cambiar en el largo plazo; por ello, para caracterizar a los estados seleccionados, sólo se consideraron, como indicadores de estudio, los porcentajes tanto de población urbana y rural como de población indígena, entre los más relevantes en México. Algunos de los factores que pueden cambiar en el corto y mediano plazo son la alfabetización, el saneamiento, los programas de salud pública y la infraestructura de los servicios de salud. Por este motivo, decidimos incluir indicadores que permitieran estimar dichos cambios en el periodo analizado ${ }^{24} \mathrm{y}$ proponer hipótesis que pudieran explicar las diferencias del impacto en los dos estados seleccionados.

Un ejemplo de intervención de impacto en el corto plazo es el Programa de Vacunación Universal. Gracias a este programa, desde 1990 se realizan actividades en forma permanente en todas las unidades del sector y se cubren jornadas intensivas durante las tres Semanas Nacionales de Salud; cada año, esta última campaña incorpora la participación de vacunadores contratados temporalmente y de numerosos voluntarios de la comunidad para aplicar una elevada proporción de vacunas. Estas acciones han incrementado y mantenido las coberturas de vacunación, inclusive en localidades sin acceso a servicios permanentes de salud, como sucede con frecuencia en Chiapas, donde la infraestructura de salud, medida por el número de médicos y enfermeras, no ha crecido lo necesario. En este sentido, la brecha que separa a este estado y a Nuevo León, no se ha acortado, sino que incluso, ha ido en aumento.

Asimismo, no obstante que la brecha en lo que se refiere a alfabetismo y saneamiento básico se acortó significativamente en el periodo estudiado, la proporción de analfabetas y de viviendas sin agua o drenaje continúa siendo elevada en Chiapas. La importancia de estos factores ha sido documentada para la enfermedad diarreica; en este caso, las estimaciones de costo-efectividad del impacto de diversas intervenciones se reflejan en el aumento del número de muertes evitadas y en la disminución del número de enfermos. ${ }^{20,25}$

En conclusión, el origen de las diferencias en el impacto de los servicios de salud, del saneamiento y de la alfabetización en los dos estados es de índole di- 
versa; entre otros aspectos, se pueden considerar los siguientes:

1. La magnitud de las tasas de mortalidad. Las más elevadas son más vulnerables que las bajas. En Chiapas, como se ha mencionado, las tasas fueron significativamente mayores, sobre todo al inicio del periodo estudiado.

2. La eficacia de las intervenciones. La vacunación es sin duda una intervención de elevada eficacia, y se ha sugerido que también puede influir en la disminución de la mortalidad por otras enfermedades infecciosas en niños ${ }_{,}^{26}$ principalmente en sitios con tasas elevadas, como es el caso de Chiapas. La reducción de la mortalidad en áreas con bajas tasas, como Nuevo León, está también relacionada con la calidad y la cobertura de los servicios médicos, con el mejor saneamiento y la mayor alfabetización.

3. La cobertura de los programas y servicios. La cobertura del Programa de Vacunación alcanzó niveles muy elevados en ambas entidades, no así la de los servicios médicos, que continúa siendo menor en Chiapas, al igual que la de agua entubada y drenaje, a pesar de los cambios registrados.

Por último, es necesario destacar que, entre 1990 y 1997, la tendencia de la mortalidad en los menores de cinco años tiene dos etapas fácilmente identificables: la primera, entre 1990 y 1992, de descenso acelerado, y la segunda, a partir de 1993, de descenso lento. Lo anterior se relaciona con los factores antes mencionados, pero fundamentalmente con el hecho, también comentado, de que las altas tasas de mortalidad como las registradas en 1990, son más vulnerables, y que es más difícil reducir tasas bajas. Sin embargo, es posible volver a acelerar la tendencia descendente. Países con tasas inferiores a las de México, algunos con economías menos desarrolladas, han logrado descensos notables. ${ }^{2}$ Para ello será necesario mantener e impulsar programas como los analizados en este trabajo, pero también será indispensable ampliar y mejorar la infraestructura de los servicios de salud. En estados con las características de Chiapas el reto es enorme.

\section{Referencias}

1. Dirección General de Coordinación Sectorial e Internacional. Estudio sectorial. La transición epidemiológica: inequidad y rezago en las condiciones de salud; diferencias regionales y estatales. México, D.F. :SSA, 1994.
2. Fondo de las $\mathrm{N}$ aciones Unidas para la Infancia. Estado Mundial de la Infancia. N ueva York: UN ICEF, 1998.

3. Consejo Nacional de Vacunación. Programas de Vacunación Universal. México, D.F.: SSA, 1990, 1995.

4. Comisión N acional de Acción en Favor de la Infancia. Programa N acional a Favor de la Infancia. México, D.F.: SSA, 1991, 1995.

5. Consejo $\mathrm{N}$ acional para la Prevención y Control de las Enfermedades Diarreicas. Programa N acional. México, D.F.: SSA, 1993.

6. Comisión N acional de Acción en Favor de la Infancia. Evaluación 1997. México, D.F.: SSA 1998.

7. Instituto Nacional de Estadística, Geografía e Informática: XI Censo General de Población y Vivienda,A guascalientes: IN EGI 1992.

8. Dirección General de Estadística e Informática. Anuarios estadísticos. México, D.F.: SSA, 1991-1997.

9. Sistema $\mathrm{N}$ acional de Vigilancia Epidemiológica. Boletines semanales 1991-1997; 8-14.

10. Consejo $\mathrm{N}$ acional de Vacunación. Encuesta Nacional de Vacunación 1990. México, D.F. :SSA, 1991.

11. Consejo N acional de Vacunación. Programa de Vacunación Universal. Coberturas. México, D.F.:SSA, 1997.

12. Instituto $N$ acional de Estadística, Geografía e Informática. Conteo de Población y Vivienda, 1995. A guascalientes: IN EGI, 1996.

13. Instituto $\mathrm{N}$ acional de Estadística, Geografía e Informática. Encuesta N acional de la Dinámica D emográfica. A guascalientes: IN EGI, 1992.

14. Consejo $\mathrm{N}$ acional del Población. Indicadores básicos de salud reproductiva y planificación familiar. México, D.F.: CO N APO, 1996.

15. Brass W, C oale A. Methods of analysis and estimation. En: BrassW, ed. The demography of Tropical Africa. Princeton: Princeton University Press, 1969.

16. Rashad H, Gray R, BoermaT. From clinical trials to large scale applications of health interventions: Facts and issues of concern. En: Rashad $H$, Gray $R$, Boerma T, ed. Evaluation of the impact of health interventions. Bélgica: International Union for the Scientific Study of Population, 1995: 1-10.

17. Kircher T, Anderson R. Cause of death. Proper completion of the death certificate. JAMA 1987;259:349-352.

18. Benavides F, Bolumar F, Peris R. Q uality of death certificates in Valencia, Spain. Am J Public Health 1989;79:1352-1354.

19. Tomé $P$, Reyes $H$, Piña $C$, Rodríguez $L$, Gutiérrez G. Características asociadas al subregistro de muerte en niños del estado de Guerrero, México. Salud Publica Mex 1997;39:523-529.

20. Gutiérrez G, Tapia R, Guiscafré $H$, Reyes H, Martínez H, Kumate J. Impact of oral rehidration and selected public health interventions on reduction of mortality from childhood diarrhoeal diseases in Mexico. Bull World Health O rgan 1996;74(2):189-197.

21. Foster S. Potential health impact of immunization. En: Rashad H, G ray $R$, Boerma T, ed. Evaluation of the impact of health interventions. Bélgica: International Union for the Scientific Study of Population, 1995.

22. Reyes $H$, Pérez-C uevas $R$, Salmerón J,Tomé $P, G$ uiscafré $H$, G Gutiérrez $G$. Infant mortaly due to acute respiratory infections: The influence of primay care processes. Health Policy Plann 1997;12(3):214-223.

23. Millard A.A causal model of high rates of child mortality. Soc Sci Med 1994; 38(2):253-268.

24. MosleyW, Chen L.An analytical framework of the study of child survival in developing countries. Pop D ev Rep 1984;10 suppl:25-45.

25. Briscoe J.W ater supply and health in developing countries: Selective primary health care revisited. Am J Public Health 1984;74(9):1009-1013. 26. Aaby P. Measles immunization and child survival: Uncontrolled experiments. En: Rashad H, Gray R, Boerma T, ed. Evaluation of the impact of health interventions. Bélgica: International Union for the Scientific Study of Population, 1995. 